\title{
BMJ Open Can earlier BCG vaccination reduce early infant mortality? Study protocol for a cluster randomised trial in Guinea- Bissau
}

\author{
Sanne M Thysen, ${ }^{\oplus 1,2,3}$ Aksel Karl Georg Jensen, ${ }^{4}$ Amabelia Rodrigues, ${ }^{3}$ \\ Igualdino da Silva Borges, ${ }^{3}$ Peter Aaby, ${ }^{2,3}$ Christine Benn, ${ }^{01,2,3}$ Ane Fisker ${ }^{1,2,3}$
}

To cite: Thysen SM, Jensen AKG, Rodrigues A, et al. Can earlier BCG vaccination reduce early infant mortality? Study protocol for a cluster randomised trial in Guinea-Bissau. BMJ Open 2019;9:e025724. doi:10.1136/ bmjopen-2018-025724

- Prepublication history and additional material for this paper are available online. To view these files, please visit the journal online (http://dx.doi. org/10.1136/bmjopen-2018025724).

Received 30 July 2018 Revised 22 August 2019 Accepted 30 August 2019

\section{Check for updates}

C Author(s) (or their employer(s)) 2019. Re-use permitted under CC BY-NC. No commercial re-use. See rights and permissions. Published by BMJ.

${ }^{1}$ OPEN, University of Southern Denmark, Odense, Denmark ${ }^{2}$ Research Center of Vitamins and Vaccines, Statens Serum Institut, Copenhagen S, Denmark ${ }^{3}$ Bandim Health Project, Bandim Health Project, Bissau, GuineaBissau

${ }^{4}$ Section of Biostatistics, University of Copenhagen, Copenhagen, Denmark

Correspondence to Dr Sanne M Thysen; s.thysen@bandim.org

\section{ABSTRACT}

Introduction The BCG vaccine is designed to protect against tuberculosis, but the vaccine may have broader effects. In 2014, the Strategic Advisory Group of Experts on Immunization reviewed the literature on non-specific effects of BCG, and concluded that the evidence was consistent with beneficial non-specific effects and requested further randomised trials.

Methods and analyses Within the Bandim Health Project's urban and rural health and demographic surveillance systems, we will conduct a clusterrandomised trial in six suburban districts and 55 rural villages. Infants are enrolled at a home visit before 72 hours of life. In intervention clusters, children are vaccinated with BCG and oral polio vaccine (OPV). In control clusters, the caregivers are informed about vaccination opportunities. Using Cox-proportional hazards models, we will test whether BCG and OPV provided at a single home visit can reduce early infant mortality up to 60 days.

The trial was initiated with a pilot study in Biombo region in June 2015. The trial was scaled up to full study including Oio and Cacheu regions in July 2016. The trial was expanded to include the urban study area in July 2017.

Ethics and dissemination BCG vaccination is delayed in many low-income settings. WHO-recommended home visits are resource demanding and vaccines are not part of the recommendation. Utilising the home visits to provide BCG and OPV may provide countries with a further incentive to introduce a single home visit. In countries, where home visits are already in place, vaccines can easily be added to reduce early infant mortality. The trial is approved by the Guinean Ethical Committee (Reference number: 0016/CNES/INASA/2015) and the Danish Ethics Committee has given its consultative approval. The results of the trial will be published in international peer-reviewed journals.

Trial registration number NCT02504203; Pre-results.

\section{INTRODUCTION}

WHO recommends home visits after birth to reduce neonatal mortality. ${ }^{12}$ Guinea-Bissau has not implemented such home visits yet. Vaccinations are not an integrated part of
Strengths and limitations of this study

- This study will provide evidence regarding the non-specific effects of BCG and oral polio vaccine (OPV) provided at birth.

- The trial will be the first randomised trial to assess non-specific effects of BCG and OPV in a rural low-income setting.

- The trial tests a feasible add-on intervention to the currently WHO-recommended home visits.

- The study will provide a cost-effectiveness analysis of providing BCG and OPV at a single home visit after birth.

- The trial tests co-administering BCG and OPV at birth and will not be able to conclude on separate non-specific effects of the individual vaccines.

home visits and are normally provided at the health centres. The current vaccination policy is to provide BCG vaccine and oral polio vaccine (OPV) at birth (vaccination schedule, figure 1).

Public health policies in low-income countries are based on a one-disease-one-solution paradigm: for each of the important diseases, a specific vaccine is developed. It is believed that the vaccine prevents only the specific disease and does nothing else. Therefore, most routine vaccines have not been tested for their effect on overall mortality.

However, many observational studies ${ }^{3-5}$ and randomised controlled trials (RCTs) ${ }^{6-9}$ in low-income countries have now shown that apart from preventing specific diseases, the vaccines may also have wider effects on the immune system, which leads to changes in resistance/susceptibility to unrelated infections. ${ }^{10}$

With respect to BCG, observational studies show that BCG is associated with better child survival, ${ }^{3} 1112$ which cannot be explained by prevention of tuberculosis (TB). ${ }^{13}$ WHO recommends BCG vaccine at birth in low-income 


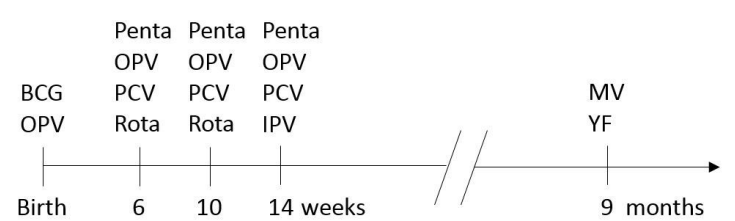

BCG: Bacillus Calmette-Guérin vaccine, OPV: Oral Polio vaccine Penta: Diphtheria, Pertussis, Tetanus, Haemophilus Influenzae B, Hepatitis B PCV: Pneumoccocal vaccine, Rota: Rotavirus vaccine, IPV: Inactivated Polio Vaccine MV: Measles vaccine, YF: Yellow Fever vaccine

Figure 1 Recommended vaccination schedule in GuineaBissau.

countries. Typically low-weight (LW) children $(<2500 \mathrm{~g})$ are only vaccinated when they reach $2500 \mathrm{~g}$. These children are assumed to be immunologically immature, and BCG vaccination is therefore often delayed. However, in three RCTs in Guinea-Bissau, BCG-at-birth reduced neonatal mortality in LW children, the combined analysis showed a mortality reduction of $38 \%$ (95\% CI: $17 \%$ to $54 \%$ ). ${ }^{67} \mathrm{~TB}$ is very rare in early childhood ${ }^{14}$ and the effects can therefore not be ascribed to prevention of TB. The effect of BCG-at-birth on neonatal mortality was seen already during the first 3 days post-vaccination, the reduction in mortality being $45 \%$ $(7 \%-68 \%) .{ }^{9}$ This rapid effect suggests that the effect is due to changes in the innate immune system.

Recent immunological studies support that BCG induces epigenetic changes in monocytes, which reprogram the innate immune system to increased pro-inflammatory responses against unrelated pathogens. ${ }^{15} 16$ These findings thus provide biological mechanisms whereby BCG could exert non-specific beneficial effects, protecting the recipients against non-targeted infectious diseases. ${ }^{16} 17$

Approximately $75 \%$ of neonatal deaths occur within the first week of life, ${ }^{13}$ but BCG is rarely given at birth in low-income countries; only $11 \%$ of the children receive BCG in the first week of life in Guinea-Bissau. ${ }^{18} \mathrm{~A}$ major reason for this delay is the focus on reducing wastage of vaccines. Freeze-dried vaccines like BCG have to be used within 6 hours after reconstitution with a diluent. Therefore, a vial of BCG vaccine is often not opened unless 10-12 children are present for vaccination. Hence, there are many missed vaccination opportunities. If BCG vaccine has profound effects on neonatal mortality and morbidity many lives could be saved by providing BCG earlier.

In this study, we aim to study the effect of BCG and OPV vaccinations provided at a single home visit within 72 hours after birth on early infant mortality and morbidity among infants in a rural and an urban setting in Guinea-Bissau. We hypothesise that BCG and OPV at birth provided at a single home visit shortly after birth reduce early infant non-accidental mortality by $40 \%$.

\section{METHODS AND ANALYSES \\ Setting}

The study is conducted in Bandim Health Project's (BHP) health and demographic surveillance system (HDSS) sites in rural and urban Guinea-Bissau.
Rural site

The rural HDSS was established in 1990. The BHP teams survey women of fertile age and their children below the age of 5 years in randomly selected clusters of villages in all the nine health regions in the country. Children in the three rural regions closest to Bissau (Oio, Biombo and Cacheu) are eligible for the study.

The rural clusters are followed with two monthly visits by the mobile teams. At all visits, the women are asked whether they are pregnant. When a pregnancy is registered, the woman's nutritional status is assessed by measurement of a mid-upper-arm-circumference (MUAC); information on antenatal care is collected prior to giving birth, as well as at the first visit after delivery. Socioeconomic factors (type of roofing, type of bathroom, possession of a mobile phone, radio and generator) are registered. After the delivery, information on the place of delivery (home, health facility) and who assisted the delivery is collected.

\section{Urban site}

The urban HDSS was established in 1978. Field assistants follow children below the age of 3 years through home visits every third month. Pregnancies are registered every month and followed monthly until birth. When a pregnancy is registered information on ethnic group, gestational age, use of mosquito net is collected. After the delivery, information on place of delivery (home, health facility) is collected. All children in the urban study area will be eligible for the study.

\section{Study nurses}

Prior to study start, we selected study nurses to conduct home visits. In the rural study area, study nurses were selected in collaboration with the regional health authorities. The nurses were selected among nurses working at a health centre with a catchment area corresponding to the study clusters, so the home visits can be done in addition to their normal routines. For the urban study area, the BHP employed a study nurse fulltime.

\section{Community key informants}

In the rural areas, to ensure that the nurses are informed immediately about deliveries, we use community key informants (CKIs). CKIs were selected among residents in each rural village to collect information about pregnancies, deliveries and deaths. The CKI communicate immediately any delivery in the village to the study nurse. In the urban area, we take advantage of the close follow-up by the field assistants, who circulate the zones daily.

\section{Study design and randomisation}

The trial is a cluster-randomised trial, randomising clusters (village clusters/sub-district zones) to two different treatment groups, stratified by region and pre-trial mortality level (high/low).

Clusters, defined as village cluster in the rural area, and as sub-district zones in the urban area, were randomised prior to study start using computer-generated random 
Table 1 Trial design

\begin{tabular}{|c|c|c|c|c|c|}
\hline Timing & $\begin{array}{l}\text { Routine visit } \\
\text { before birth }\end{array}$ & \multicolumn{2}{|c|}{ Shortly after birth - within 72 hours } & $\begin{array}{l}\text { First routine visit after } \\
\text { birth }\end{array}$ & $\begin{array}{l}\text { Second and third } \\
\text { routine visit after birth }\end{array}$ \\
\hline Personnel & $\begin{array}{l}\text { BHP field assistant } \\
\text { and nurse }\end{array}$ & CKI & Study nurse & $\begin{array}{l}\text { BHP field assistant } \\
\text { and nurse }\end{array}$ & $\begin{array}{l}\text { BHP field assistant } \\
\text { and nurse }\end{array}$ \\
\hline Control group & $\begin{array}{l}\text { Information about } \\
\text { trial and invitation } \\
\text { to participate. } \\
\text { Informed consent. }\end{array}$ & $\begin{array}{l}\text { CKI inform about } \\
\text { birth }\end{array}$ & $\begin{array}{l}\text { Umbilical cord and } \\
\text { skin care } \\
\text { Encourage skin-to- } \\
\text { skin contact to keep } \\
\text { the newborn warm } \\
\text { Examine and weigh } \\
\text { the child } \\
\text { Inform about } \\
\text { vaccination } \\
\text { opportunities }\end{array}$ & $\begin{array}{l}\text { Field assistant: } \\
\text { Interview on } \\
\text { morbidity and } \\
\text { mortality } \\
\text { Nurse: } \\
\text { Examine the BCG } \\
\text { vaccination site } \\
\text { and lymph glands } \\
\text { Measure } \\
\text { temperature } \\
\text { Weigh the child } \\
\text { Provide BCG and } \\
\text { OPV }\end{array}$ & $\begin{array}{l}\text { Field assistant: } \\
\text { Interview on } \\
\text { morbidity and } \\
\text { mortality } \\
\text { Nurse: } \\
\text { Examine the BCG } \\
\text { vaccination site } \\
\text { and lymph glands } \\
\text { Measure } \\
\text { temperature } \\
\text { Weigh the child } \\
\text { Provide routine } \\
\text { vaccinations }\end{array}$ \\
\hline
\end{tabular}

Bold and italics stress the differences between the intervention and control groups.

BHP, Bandim Health Project; CKI, community key informant; OPV, oral polio vaccine.

numbers. All newborns are visited as soon as possible after birth. In half of the clusters, we provide standard care and vaccines (BCG, OPV) to children at the home visits; in the remaining clusters, children only receive standard care but no vaccines (table 1 ). The trial is unblinded.

\section{Inclusion criteria}

All children registered during pregnancy are eligible for the study. Children can only be enrolled if they are visited within 72 hours after birth.

\section{Exclusion criteria}

There are few exclusion criteria, because the study is expected to answer a pragmatic question about the effect of BCG and OPV vaccination at home visits shortly after birth.

- Children already BCG vaccinated.

- Moribund children (expected not to survive the next 24 hours, as evaluated by the study nurse at the enrolment visit).

- Children in rural villages where the BHP mobile teams coincidentally were in the village the same day (and vaccinated all children).

\section{Informed consent}

All pregnant women receive an oral and a written explanation of the study, and are offered to participate in the study at the time of registration of the pregnancy. In the rural area, the explanation is given by the BHP nurse at the two monthly visits. In the urban area, the explanation is given by the fieldwork assistant at a monthly visit. It is explained that normally newborn children receive their first vaccinations at the health centres. However, studies have suggested that it might be beneficial to receive BCG and OPV early and the BHP will therefore test the effect of providing the vaccines at home visits. All children receive a home visit with standard care. Children in half the clusters (intervention clusters) receive BCG and OPV at enrolment. Children in the control clusters can seek vaccination elsewhere and will be offered the BCG vaccine and OPV at the next home visit by the BHP team if they did not get it elsewhere. If the pregnant woman accepts to take part in the study, they are asked to sign or fingerprint a consent form. An independent witness confirms the consent process by co-signing the form.

\section{Enrolment}

The study nurse visits every newborn child shortly after a $\mathrm{CKI} /$ mother calls, if possible on the same day. At the 
home visit, the nurse asks the mother for confirmation of her consent for participation in the study before revealing the randomisation. If the mother is not able to present the signed consent form, new consent forms are filled in. The nurse brings a questionnaire, a sticker with study number and a vaccination card.

\section{Intervention}

For all children, the nurse examines and weighs the child, encourages skin-to-skin contact to keep the newborn warm and if necessary performs umbilical cord care. ${ }^{1}$ Following interview and examination, group allocation is disclosed to the mother. For children in the control clusters, the nurse informs about vaccination opportunities (vaccination at closest health centre or vaccination by BHP nurse at next visit), as recommended by WHO. ${ }^{1}$ For children in the intervention clusters, the nurse administers BCG and OPV (table 1).

\section{BCG vaccine}

BCG vaccination is administered by intradermal injection; after vaccination most children develop a scar at the injection site. Among BCG-vaccinated children, having a BCG scar is associated with improved survival. ${ }^{19-21}$ The proportion of children developing a scar after BCG vaccination depends on the vaccination technique and strain. ${ }^{22-24}$ All nurses have been trained prior to the trial and supervised intensively during the beginning of the trial to ensure correct vaccination technique. The Tokyo 172 BCG strain from BCG Japan is used for the study. The vaccine is prequalified by $\mathrm{WHO}$ and is regularly used in the national vaccination programme in Guinea-Bissau.

\section{OPV vaccine}

The OPV vaccine is supplied from the national vaccination programme and thus strain, manufacturer and batch may vary. For all vaccines, the manufacturer, batch and expiration date are registered.

\section{Follow-up}

All children enrolled in the study receive two follow-up visits. At follow-up visits, the child is weighed, and the BHP nurse examines lymphadenopathy and reaction at the vaccination site.

\section{Follow-up in the rural regions}

The study participants are followed through the rural HDSS, where all children below 5 years of age are followed, and information on vital status, breastfeeding status, supplementary feeding, MUAC, vaccinations, hospital admissions and whether the child has received interventions provided in campaigns is collected.

The BHP teams are accompanied by a nurse, who administers vaccines. The BHP nurse performs the follow-up visits for this study. All children have their vaccination cards examined and those missing one or more routine vaccines according to the schedule are offered these vaccines at the follow-up visits.

\section{Follow-up in the urban region}

The study participants receive two follow-up visits by the study nurse at 2 and 4 months of age. The study nurse brings BCG and OPV for children in control clusters. For the remaining routine vaccines as part of the national programme, the children are referred to the closest health centre. All children below the age of 3 years are followed through home visits every third month, where information on vital status, breastfeeding status, supplementary feeding, MUAC, vaccinations, hospital admissions and vaccination campaigns is collected.

\section{Outcomes}

The primary outcome is non-accidental mortality rate between enrolment visit or 24 hours after birth, whichever comes last, and 60 days of life. Follow-up time will be censored at the first of visit by the BHP, death due to accident, date of registration of first non-trial vaccine given after enrolment or migration. All children living in intervention and control clusters are followed through the HDSS routines with collection of information on vital status for all children. Deaths are classified as accidental or non-accidental based on information from verbal autopsies.

\section{Secondary outcomes and potential effect modifiers}

We will evaluate the effect on severe morbidity considered as the composite outcome of non-accidental death and non-accidental hospital admission. With declining mortality, we may not be able to obtain a conclusive result for the primary outcome. We have defined the sample size for the composite outcome, but retained mortality as the primary outcome.

We will conduct secondary analyses to assess whether an effect varies by low birth weight $(<2500 \mathrm{~g})$, sex, maternal BCG scarring and season to gain a better knowledge of the potential effect modifiers (a list of all outcomes can be found in box 1).

We will study the cost-effectiveness of providing BCG and OPV at a home visit using the effects on mortality and hospital admission from this project.

For all deaths a verbal autopsy will be made. These will mainly be used to classify a death as accidental or non-accidental, but where possible, we will also report cause-specific mortality.

\section{Data management}

All data are collected on paper forms and entered in the BHP office. Records with registration of all pregnant women are copied from the data tables of the routine HDSS to the BCG trial data table. Forms for follow-up are pre-printed with identification information of the enrolled children. For routine HDSS visits, a list of all pregnant women is printed prior to the visit to ensure that all pregnant women are invited to participate in the study prior to giving birth. Following data entry, the data are checked for consistency. Inconsistencies between the 
Box 1 Outcomes

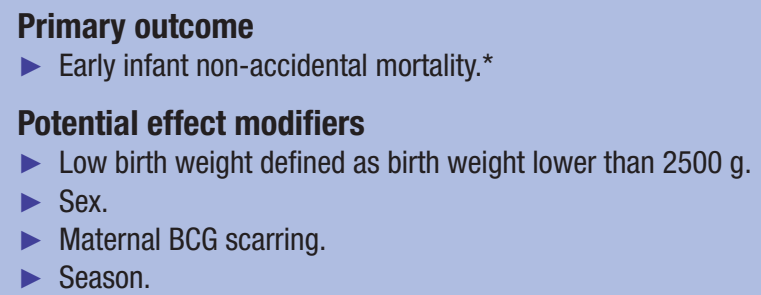

Primary outcome

- Early infant non-accidental mortality. *

Potential effect modifiers

- Low birth weight defined as birth weight lower than $2500 \mathrm{~g}$.

- Sex.

- Maternal BCG scarring.

Season.

\section{Secondary outcomes}

- Non-accidental hospital admission.*

- Severe morbidity (composite outcome of non-accidental mortality and non-accidental hospital admissions). ${ }^{*}$

- Consultations. $\dagger$

- Growth. $\neq$

Mid-upper-arm circumference.

Weight-for-age z-score.

- BCG scarring. $\dagger$

- Cost-effectiveness of providing BCG and oral polio vaccine at home visits.

- Cause-specific mortality.

*Analysed using Cox proportional hazards models. Further details are provided in online supplementary appendix 2.

†Analysed using log-binomial regression. Further details are provided in online supplementary appendix 2.

$\ddagger$ Analysed using linear regression. Further details are provided in online

supplementary appendix 2.

BCG trial data and the HDSS data are checked. Main outcome events are reviewed individually.

\section{Statistical analyses}

General analysis principles applied in all analyses can be found in online supplmentary appendix 1. In Cox-proportional hazards models with age as underlying timescale, we will assess the effect of providing BCG and OPV at a single home visit. We will allow for different baseline hazards according to the factors used in the randomisation (region and pre-study mortality level) and sex. Children enter the analysis on the date of enrolment or 24 hours of age, whichever comes last, and remain in the analysis until the first subsequent visit by the BHP team, date of registering first non-trial vaccine after enrolment, death or migration within the first 60 days of life. In secondary analyses, we will investigate whether the effect of BCG +OPV differ by the following potential effect modifiers, which in prior trials have been important determinants of the effect: low birth weight $(<2500 \mathrm{~g})$, sex, maternal BCG scarring, and season. Further details are provided in online supplementary appendix 2.

Secondary outcomes are non-accidental hospital admissions, severe morbidity (composite outcome of non-accidental mortality and non-accidental hospital admissions), consultations, growth (MUAC and weight-for-age z-score) and BCG scarring. Analyses are described in online supplementary appendix 2 . We will furthermore perform a cost-effectiveness analysis seeking to measure the cost per death averted using a societal perspective, contrasting the costs of vaccine provision in the present programme and an outreach system as tested in the trial. The final analysis plan is deposited with the Data Safety and Monitoring Board. A list of all planned analyses is provided in online supplementary appendix 2.

\section{Sample size considerations}

We estimated the design effect to 1.35 (ratio of square of the standard errors for the cluster-adjusted/unadjusted Hazard Ratios (HR)) based on pre-trial event data (deaths and hospitalisation) from the rural HDSS clusters, where the study is conducted. We base our sample size calculation on the combined secondary outcome for feasibility reasons, but retain mortality as the primary outcome. We estimated the proportion of events to be $2.4 \%$ in the absence of our intervention (prior data). Thus, in order to obtain $80 \%$ power to detect a reduction in early infant severe morbidity if the true reduction of BCG and OPV provided at home visits is larger than $40 \%$, we will need to enrol at least 6666 children. This sample size has $74 \%$ power to detect a significant difference in mortality if the real effect is a $40 \%$ reduction from a mortality level of $2.1 \%$.

\section{Time schedule}

The trial was initiated with a pilot study in Biombo region in June 2015. The trial was scaled up to full study including Oio and Cacheu regions in July 2016. The trial was expanded to include the urban study area in July 2017. In March 2019, the trial was stopped in the urban study area and in the Cacheu region, due to lower than anticipated number of enrolments and number of events, resulting in a lower than anticipated power. As a result of the lower than anticipated power, we plan to end the trial prematurely in Oio and Biombo health regions in September 2019. The results of the trial will be reported as planned despite the lower than anticipated power.

\section{Ethics and dissemination}

\section{Ethical considerations}

BCG is recommended at birth but vaccination is often delayed; in rural Guinea-Bissau most children receive BCG after 1 month. ${ }^{18}$ While there is now increasing acceptance that BCG may have beneficial non-specific effects, we are still a long way from altering the implementation of the vaccination programme. The proposal compares two ways of delivering BCG and OPV; both are an improvement relative to the current situation in rural Guinea-Bissau, where less than $40 \%$ of all infants get BCG during the first month of life. ${ }^{18}$ Hence, no child receives BCG later than it would have done, had the trial not been carried out. The trial is registered at clinicaltrials.gov.

\section{Safety monitoring}

A data safety and monitoring board has been formed consisting of a paediatrician (Anja Poulsen, Rigshospitalet, Denmark), a statistician (Morten Frydenberg, Aarhus University, Denmark) and an epidemiologist (Torben Sigsgaard, Aarhus University, Denmark). The 
members have been appointed on their experience, reputation for objectivity, absence of conflicts of interest and knowledge on clinical trial methodology.

The vaccines used for the trial are prequalified by WHO to be given at birth. Adverse reactions are rare for both BCG and OPV. At the two first visits after enrolment in the study, the BHP nurse examines the BCG vaccination site and the axillary lymph glands of all children to assess suppurative lymphadenitis as an adverse reaction to the BCG vaccination. Other serious adverse events are captured through primary and/or secondary outcomes (mortality, hospitalisations and consultations).

\section{Dissemination}

The results of the study will be published in international peer-reviewed journals and results will be communicated to the National Institute of Public Health in Guinea-Bissau. After publication of the main results on completion of the trial, data requests can be submitted to the researchers at BHP.

The SPIRIT checklist can be found in the online supplementary file 1 .

\section{Protocol amendments}

Any protocol modifications will be discussed with the Data Safety and Monitoring Board, and changes will be added to the trial registration.

\section{Patient and public involvement}

The communities were involved in locating households, when the HDSS was setup and contributed information allowing tracing of internal migrants between villages throughout the study period. No participants were involved in setting the research question or the outcome measure, nor were they involved in developing plans for recruitment, design or implementation of the study. Local CKIs were selected to ensure timely information about birth. Nurses from local health centres are involved in the study and have been trained to perform enrolment. This ensures a close collaboration with the local health system. No participant was asked to advise on interpretation or writing up the results. The results are disseminated to the national public health institute. There are no plans to disseminate the results of the research to study participants. Study results will be disseminated to local nurses involved in the study.

\section{DISCUSSION}

WHO recommends BCG and OPV at birth. However, due to the focus on not wasting vaccine doses, BCG vaccination is often delayed. ${ }^{18}{ }^{25-27}$ Previous trials from Guinea-Bissau have showed that early vaccination may have major impact on neonatal mortality among LW children, ${ }^{679}$ and observational studies have suggested that BCG also has beneficial non-specific effects(NSE) among normal-birth-weight children. ${ }^{311}$ In 2014, the Strategic Advisory Group of Experts on Immunization reviewed the non-specific effects of the
BCG vaccine, and concluded that the evidence was consistent with beneficial NSEs. However, the bulk of evidence came from observational studies and they therefore called for randomised trials. To our knowledge, this will be the first randomised trial assessing the NSEs of BCG and OPV vaccination among children in a rural low-income setting. The SAGE review did not include OPV, but a recent trial from Guinea-Bissau randomised children to BCG only or BCG and OPV, and found that OPV was associated with a $32 \%$ reduction in mortality. ${ }^{28}$ Thus, both BCG and OPV may have beneficial NSEs. The results will therefore be very important in broadening our understanding of the NSEs of BCG and OPV at birth. We will however not be able to distinguish the effects of BCG and OPV in this trial.

WHO recommends three home visits after birth, which is resource demanding and not implemented in many low-income countries. It is not recommended that the home visits are utilised for early vaccination. However, we hypothesise that a single home visit after birth will have major effects on early infant mortality if BCG and OPV vaccination are added to the home visit. If BCG and OPV provided at a single home visit can reduce early infant mortality, this should be included in the WHO recommendations. A single home visit with vaccines will be easier to implement in a strained health system than three home visits within 7 days. In countries, where home visits are already in place, vaccines can easily be added to reduce early infant mortality.

Acknowledgements We thank our Data Safety and Monitoring Board, Anja Poulsen, Morten Frydenberg and Torben Sigsgaard, for insightful comments on planned analysis, study design and implementation.

Contributors AR, PA and CB conceived the idea for the trial. SMT, PA, CB and AF designed the study. SMT set up the study with help from ABF. SMT, IdSB and AF supervised data collection. SMT, AKGJ, and AF planned the data analyses. SMT drafted the manuscript and data analysis plan with help from AKGJ and AF. All authors read and approved the final manuscript and data analysis plan.

Funding Karen Elise Jensen Foundation is the main funder of the trial. Laerdal Foundation supports the trial. The Research Center for Vitamins and Vaccines, Bandim Health Project receives support from the Danish National Research Foundation (DNRF108). The funders had no role in the design of the study or writing of the protocol.

Competing interests None declared.

Patient consent for publication Not required.

Provenance and peer review Not commissioned; externally peer reviewed.

Open access This is an open access article distributed in accordance with the Creative Commons Attribution Non Commercial (CC BY-NC 4.0) license, which permits others to distribute, remix, adapt, build upon this work non-commercially, and license their derivative works on different terms, provided the original work is properly cited, appropriate credit is given, any changes made indicated, and the use is non-commercial. See: http://creativecommons.org/licenses/by-nc/4.0/.

\section{REFERENCES}

1. WHO, UNICEF. WHO/Unicef joint statement: home visits for the newborn child: a strategy to improve survival, 2009.

2. WHO. Who recommendations on postnatal care of the mother and newborn, 2013. Available: http://apps.who.int/iris/bitstream/handle/ 10665/97603/9789241506649_eng.pdf?sequence $=1$

3. Kristensen I, Aaby P, Jensen H. Routine vaccinations and child survival: follow up study in Guinea-Bissau, West Africa. BMJ 2000;321:1435-8. 
4. Aaby P, Benn C, Nielsen J, et al. Testing the hypothesis that diphtheria-tetanus-pertussis vaccine has negative non-specific and sex-differential effects on child survival in high-mortality countries. BMJ Open 2012;2:e000707.

5. Aaby P, Ravn H, Roth A, et al. Early diphtheria-tetanus-pertussis vaccination associated with higher female mortality and no difference in male mortality in a cohort of low birthweight children: an observational study within a randomised trial. Arch Dis Child 2012;97:685-91.

6. Aaby P, Roth A, Ravn H, et al. Randomized trial of BCG vaccination at birth to low-birth-weight children: beneficial nonspecific effects in the neonatal period? J Infect Dis 2011;204:245-52.

7. Biering-Sørensen S, Aaby P, Napirna BM, et al. Small randomized trial among Low-birth-weight children receiving Bacillus CalmetteGuéerin vaccination at first health center contact. Pediatr Infect Dis $J$ 2012;31:306-8.

8. Aaby P, Jensen H, Samb B, et al. Differences in female-male mortality after high-titre measles vaccine and association with subsequent vaccination with diphtheria-tetanus-pertussis and inactivated poliovirus: reanalysis of West African studies. The Lancet 2003;361:2183-8

9. Biering-Sørensen S, Aaby P, Lund N, et al. Early BCG-Denmark and neonatal mortality among infants weighing $<2500 \mathrm{~g}$ : a randomized controlled trial. Clin Infect Dis 2017;65:1183-90.

10. Benn CS, Netea MG, Selin LK, et al. A small jab - a big effect: nonspecific immunomodulation by vaccines. Trends Immunol 2013;34:431-9.

11. Hirve S, Bavdekar A, Juvekar S, et al. Non-specific and sexdifferential effects of vaccinations on child survival in rural Western India. Vaccine 2012;30:7300-8.

12. Aaby P, Kollmann TR, Benn CS. Nonspecific effects of neonatal and infant vaccination: public-health, immunological and conceptual challenges. Nat Immunol 2014;15:895-9.

13. Lawn JE, Cousens S, Zupan J 4 million neonatal deaths: when? where? why? The Lancet 2005;365:891-900.

14. Shann F. Commentary: BCG vaccination halves neonatal mortality. Pediatr Infect Dis J 2012;31:308-9.

15. Netea MG, Quintin J, van der Meer JWM. Trained immunity: a memory for innate host defense. Cell Host Microbe 2011;9:355-61.

16. Kleinnijenhuis J, Quintin J, Preijers F, et al. Bacille Calmette-Guerin induces Nod2-dependent nonspecific protection from reinfection via epigenetic reprogramming of monocytes. Proc Natl Acad Sci U S A 2012;109:17537-42.

17. Jensen KJ, Larsen N, Biering-Sørensen S, et al. Heterologous immunological effects of early BCG vaccination in low-birth-weight infants in Guinea-Bissau: a randomized-controlled trial. J Infect Dis 2015;211:956-67.

18. Thysen SM, Byberg S, Pedersen M, et al. Bcg coverage and barriers to BCG vaccination in Guinea-Bissau: an observational study. BMC Public Health 2014;14:1037.

19. Roth A, Gustafson P, Nhaga A, et al. Bcg vaccination scar associated with better childhood survival in Guinea-Bissau. Int $J$ Epidemiol 2005;34:540-7.

20. Roth A, Sodemann M, Jensen $\mathrm{H}$, et al. Tuberculin reaction, BCG scar, and lower female mortality. Epidemiology 2006;17:562-8.

21. Garly M-L, Martins CL, Balé C, et al. Bcg scar and positive tuberculin reaction associated with reduced child mortality in West Africa. A non-specific beneficial effect of BCG? Vaccine 2003;21:2782-90.

22. Roth $A$, Sodemann $M$, Jensen $H$, et al. Vaccination technique, PPD reaction and BCG scarring in a cohort of children born in GuineaBissau 2000-2002. Vaccine 2005;23:3991-8.

23. Frankel $\mathrm{H}$, Byberg $\mathrm{S}$, Bjerregaard-Andersen $\mathrm{M}$, et al. Different effects of BCG strains - a natural experiment evaluating the impact of the Danish and the Russian BCG strains on morbidity and scar formation in Guinea-Bissau. Vaccine 2016;34:4586-93.

24. Funch KM, Thysen SM, Rodrigues A, et al. Determinants of BCG scarification among children in rural Guinea-Bissau: a prospective cohort study. Hum Vaccin Immunother 2018;14:2434-42.

25. Kagoné $\mathrm{M}, \mathrm{Ye} \mathrm{M}$, Nébié $\mathrm{E}$, et al. Vaccination coverage and factors associated with adherence to the vaccination schedule in young children of a rural area in Burkina Faso. Glob Health Action 2017; 10:1399749.

26. Hanifi SMA, Das S, Rahman M. Bangladeshi neonates miss the potential benefits of early BCG vaccination. Int $\mathrm{J}$ Epidemio 2018;47:348-9

27. Wallace AS, Willis F, Nwaze E, et al. Vaccine wastage in Nigeria: an assessment of wastage rates and related vaccinator knowledge, attitudes and practices. Vaccine 2017;35:6751-8.

28. Lund N, Andersen A, Hansen ASK, et al. The effect of oral polio vaccine at birth on infant mortality: a randomized trial. Clin Infect Dis 2015;61:1504-11. 